\title{
Catalytic Radical-Polar Crossover Ritter Reaction
}

\author{
Eric E. Touney ${ }^{\star}$, Riley E. Cooper ${ }^{\ddagger}$, Sarah. E. Bredenkamp, David T. George ${ }^{\dagger}$, and Sergey V. Pronin* \\ Department of Chemistry, University of California, Irvine, California 92697-2025, United States
}

Supporting Information Placeholder

\begin{abstract}
A catalytic radical-polar crossover Ritter reaction is described. The transformation proceeds under acid-free conditions and tolerates a variety of functional groups. The catalyst design overcomes limitations in the substitution pattern of starting materials and enables hydroamidation of a diverse range of alkenes. Formation of hydrogen contributes to the background consumption of reductant and oxidant and competes with the desired pathway, pointing to a mechanistic link between hydrogen atom transfer-initiated organic reactions and hydrogen evolution catalysis.
\end{abstract}

Hydroamidation of alkenes with nitriles in the presence of a strong Brønsted acid, discovered by Ritter, allows for a convenient access to tert-alkylamines and their derivatives from simple precursors. ${ }^{1}$ The Ritter reaction is used in production of tert-octylamine and its higher molecular weight homologs. ${ }^{2}$ Variants of the Ritter reaction have found application in pharmaceutical industry and academic research for installation of nitrogen-based functionalities. ${ }^{3,4}$ The rate-determining protonation of the carbon-carbon double bond necessitates forcing conditions and application of strong Brønsted acids, which results in limited functional group tolerance. Here we demonstrate an acid-free Ritter reaction enabled by cobalt-catalyzed radical-polar crossover hydroamidation of alkenes. This transformation takes advantage of hydrogen atom transfer (HAT) followed by oxidation to generate the corresponding carbocationic intermediates, accomplishing formal protonation of the carbon-carbon double bond and providing excellent tolerance of functional groups (Scheme 1). We show that catalysis by modified cobalt salen complexes overcomes limitations in the substitution pattern of the alkenes and allows for hydroamidation of a diverse range of substrates. We also present data indicating that the mechanism of background consumption of reductant and oxidant includes formation of hydrogen, which competes with the desired pathway, explaining prior limitations in the substrate scope.

During our investigations into HAT-initiated radical-

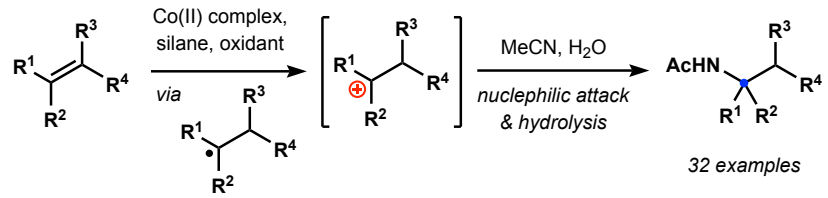

Scheme 1. Catalytic radical-polar crossover Ritter reaction.

polar crossover reactions of alkenes we observed that application of acetonitrile as solvent occasionally resulted in formation of small amounts of side-products arising from apparent Markovnikov addition of acetamide to the carbon-carbon double bond. ${ }^{5,6}$ The electrophilic intermediates in this formal Ritter reaction were proposed to arise from direct oxidation of alkyl radicals following the HAT and diffusion from the solvent cage. ${ }^{7}$ Alternative pathways involve radical pair collapse to generate the corresponding alkylcobalt(III) complexes, which undergo oxidation to alkylcobalt(IV) species and subsequent nucleophilic displacement or dissociation to the carbocations (Scheme 2). ${ }^{8-10}$ Development of the radical-polar cross-

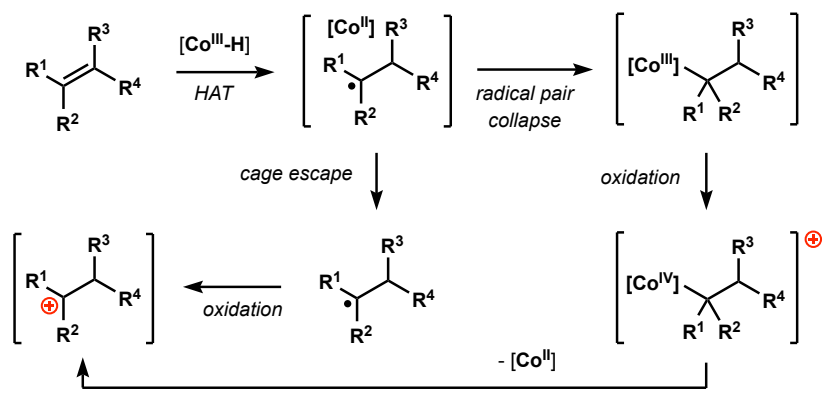

Scheme 2. Pathways for generation of electrophilic intermediates in HAT-initiated radical-polar crossover reactions.

over variant of the Ritter reaction is appealing because it is expected to improve the poor functional group tolerance associated with the application of strong Brønsted acids. Similar benefits were demonstrated in the previous HAT-initiated hydrofunctionalizations to install nitrogenbased functionalities, including landmark reports of radical hydrohydrazidation and hydroazidation reactions and more recent radical-polar crossover hydroazolation and intramolecular hydroamidation methods (Scheme 3). ${ }^{11-16}$ 
- Hydrohydrazidation (ref. 11,13)

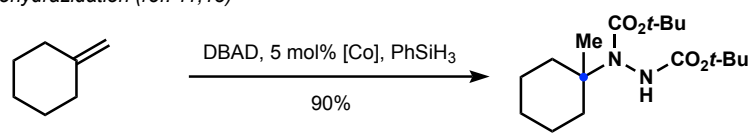

- Hydroazidation (ref. 12,13)

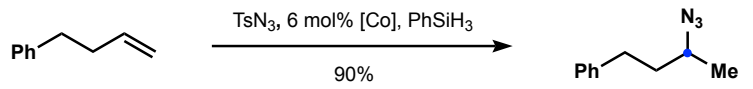

- Intramolecular hydroamidation (ref. 15)

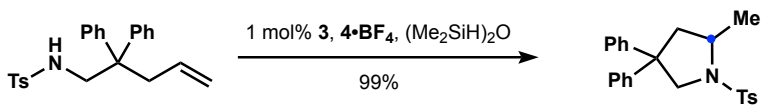

- Hydroazolation (ref. 16)
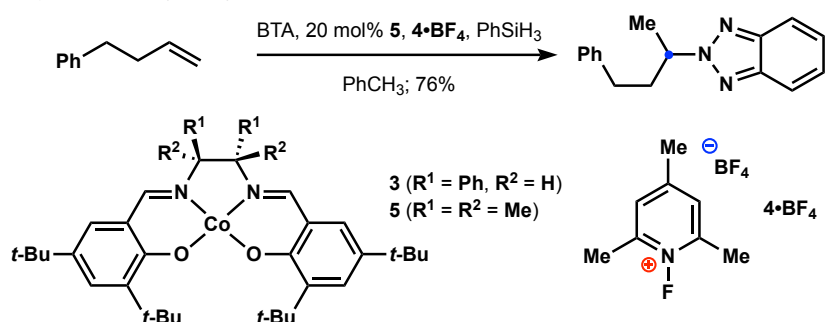

Scheme 3. Examples of relevant hydrofunctionalizations.

Our initial optimization efforts allowed for efficient conversion of dihydrocarvone to the corresponding hydroamidation product in the presence of catalyst $\mathbf{5}$ (Scheme 4). At the same time, we observed significant<smiles>C=C(C)C1CCC(C)C(=O)C1</smiles>

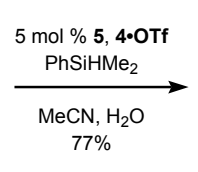
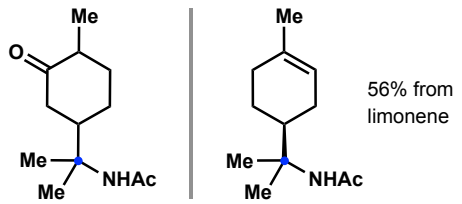

Scheme 4. Initial studies in the catalytic radical-polar crossover Ritter reaction.

limitations in the substitution pattern at the carbon-carbon double bond with only 1,1-disubstituted alkenes showing optimal performance. As a corollary, these limitations could be leveraged to achieve selective Ritter reactions of differentially substituted polyenes, such as limonene. Notably, similar challenges were previously associated with other relevant radical-polar crossover hydrofunctionalizations and also highlighted in a recent study of HATinitiated hydroamidation of alkenes. ${ }^{15,17}$ Here we overcome these limitations using catalyst design and relate the substrate intolerance to competing hydrogen evolution.

To address the identified limitations in the scope of alkenes, we focused our efforts on substrates that appeared inert towards reaction conditions that successfully engaged 1,1-disubstituted alkenes in the HAT-initiated Ritter reaction. Thus, attempted hydroamidation of fully substituted alkene $\mathbf{6}$ in the presence of catalyst 5 did not induce any significant reactivity of the substrate (Table 1). Application of complexes $\mathbf{3}$ and $\mathbf{9}$, commonly employed in other HAT-initiated reactions, produced a mixture of amides $\mathbf{7}$ and $\mathbf{8}$, but returned the majority of material in the form of starting alkene 6. Similar outcome was observed in the presence of complex $\mathbf{1 0}$, which contained
Table 1. Effect of the Catalyst Structure on the Catalytic Radical-Polar Crossover Ritter Reaction of Alkene $6^{\text {a }}$

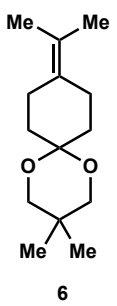

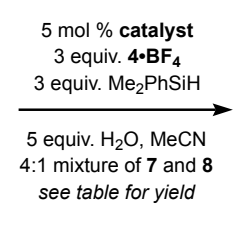

catalyst 5

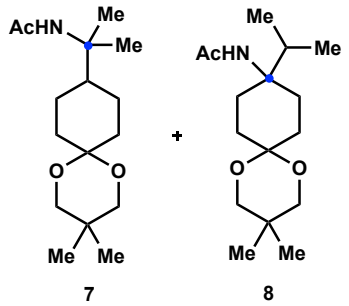

$0 \%$

$34 \%$

9: $40 \%$
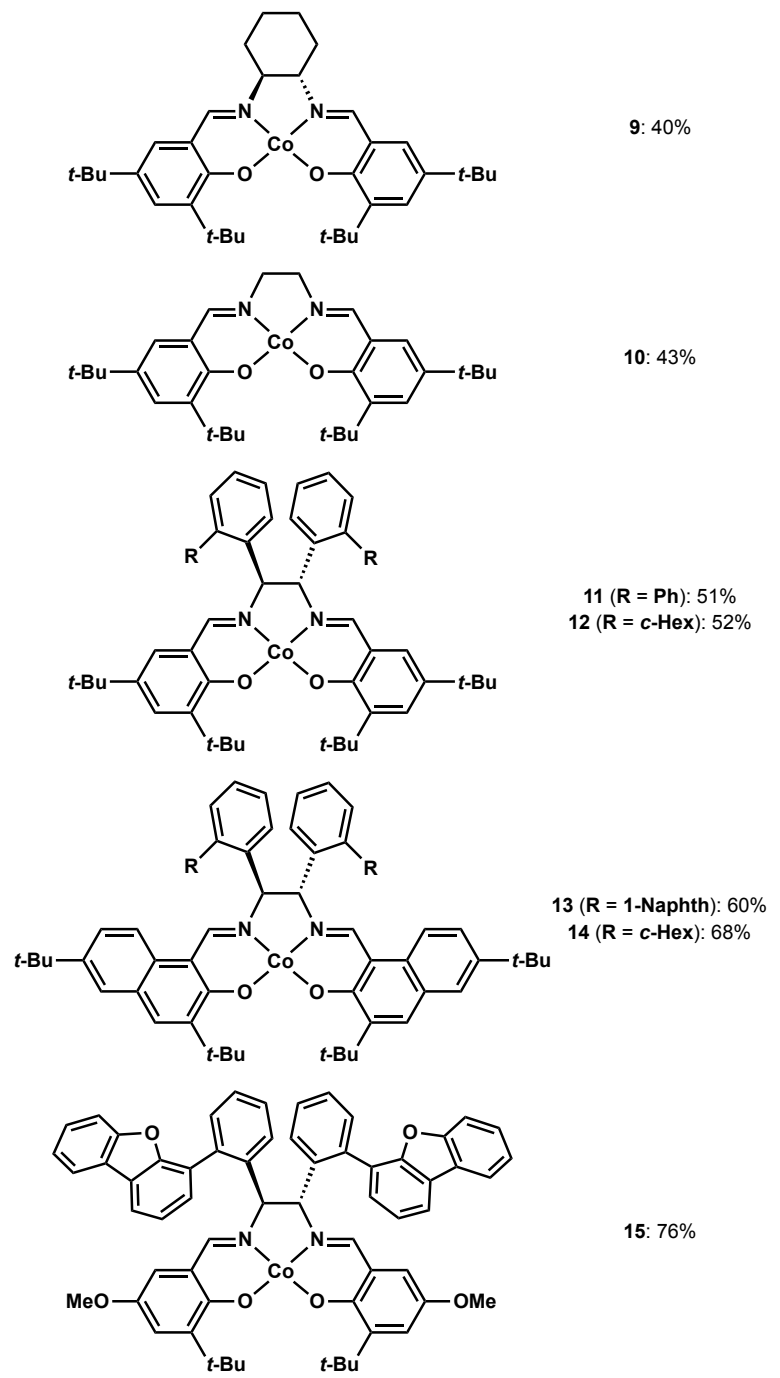

${ }^{a}$ yields and ratios were determined by ${ }^{1} \mathrm{H}$ NMR analysis. Isolated yield with catalyst 15 was $76 \%$.

unsubstituted ethylenediamine motif. Increased reaction times, additional amounts of the oxidant and reductant, and changes in the order of addition did not lead to improved outcomes. ${ }^{18}$ Subsequent modifications in the salen ligand were more fruitful and produced several instructive trends. Thus, application of complexes containing $O$ substituted aromatic motifs in the ethylenediamine-derived fragment delivered improved yields of products 7 and $\mathbf{8}$, as seen in the cases of catalysts $\mathbf{1 1}$ and $\mathbf{1 2}$. 
Table 2. Preliminary Substrate Scope of the Catalytic Radical-Polar Crossover Ritter Reaction ${ }^{\text {a }}$

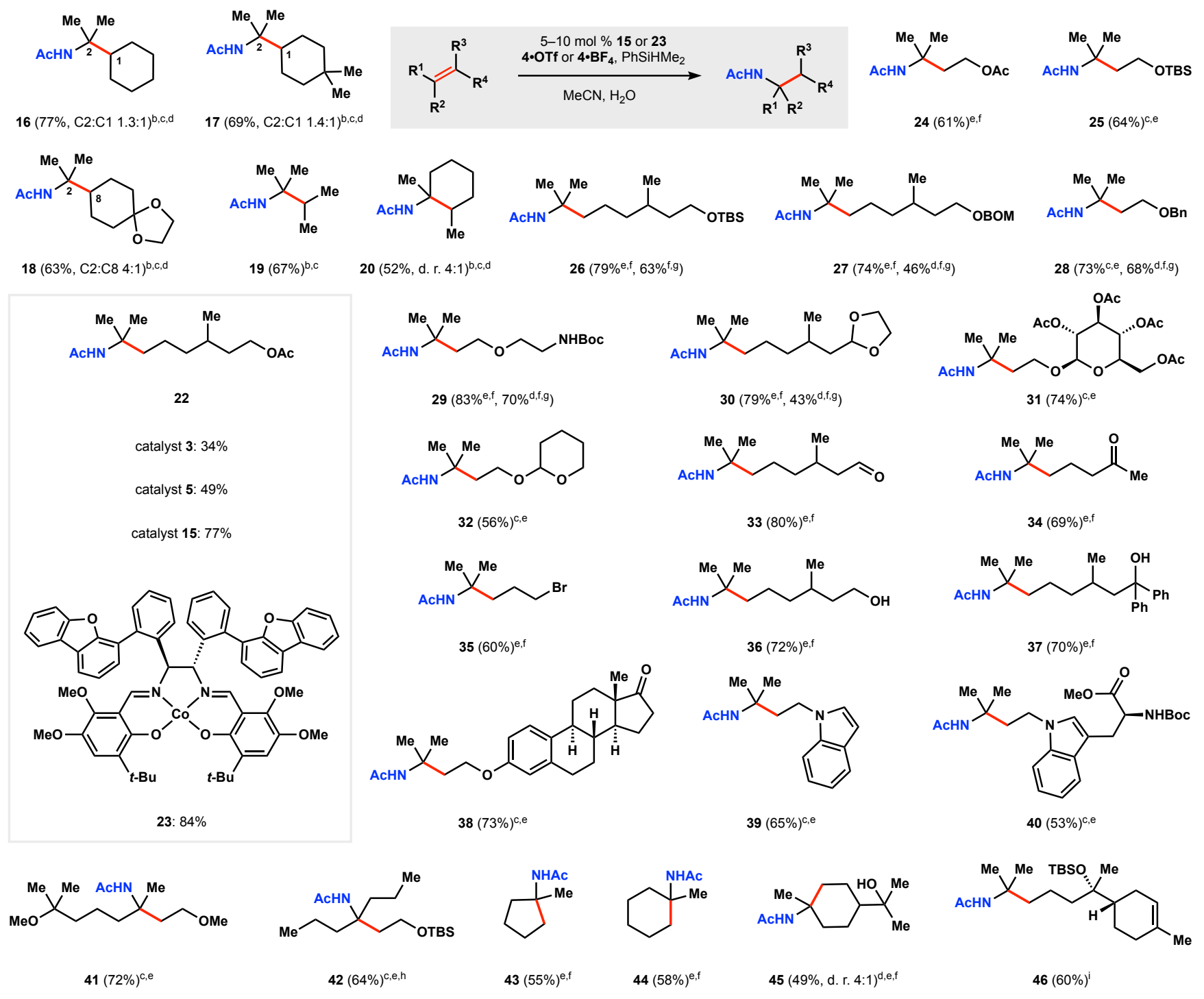

${ }^{a}$ unless noted otherwise, reactions were conducted with $5 \mathrm{~mol} \%$ of catalyst, 3 equiv. of oxidant and silane, and 5 equiv. of water. Yields in parentheses correspond to isolated, analytically pure material. ${ }^{b}$ with catalyst 15 . ${ }^{c}$ with $\mathbf{4} \cdot \mathbf{B F} 4 .{ }^{d}$ determined by ${ }^{1} \mathrm{H}$ NMR analysis. ${ }^{\mathrm{e}}$ with catalyst 23. ${ }^{\mathrm{f}}$ with $\mathbf{4} \cdot$ OTf. ${ }^{\mathrm{g}}$ with catalyst 5 . ${ }^{\mathrm{h}}$ with 3 equiv. of water. ${ }^{\mathrm{i}}$ with $10 \mathrm{~mol} \%$ of catalyst, 6 equiv. of silane and oxidant, and 10 equiv. of water.

Introduction of $o$-aryl and $o$-cyclohexyl substituents led to similar outcomes, suggesting that the effect was largely steric in nature. At the same time, presence of extended aromatic motifs in the salicylaldehyde-derived fragment was beneficial, and good yields of the hydroamidation products were obtained with catalyst 13 and $\mathbf{1 4}$. Ultimately, complex 15 containing an electron-rich salen motif provided a somewhat improved performance among the evaluated catalysts. In all cases, amides $\mathbf{7}$ and $\mathbf{8}$ were formed in the same ratio, accompanied by small amounts of the hydrogenation and hydration products. ${ }^{19-20}$

Brief evaluation of different tetrasubstituted alkenes revealed suitability of our protocol for generation of the corresponding Ritter products. Exocyclic alkenes related to compound $\mathbf{6}$ underwent hydroamidation in similar yields and produced mixtures of regioisomeric amides (products
16-18, Table 2). Application of simple acyclic and cyclic tetrasubstituted alkenes was comparably successful (products 19 and 20). Citronellyl acetate (21) also underwent efficient hydroamidation under the optimized conditions (product 22). Brief assessment of the catalyst effect in reactions with alkene 21 re-affirmed superior performance of electron-rich salen motifs containing $o$ biaryl substituents in the ethylenediamine-based fragment, where catalyst $\mathbf{2 3}$ offered slight improvement over complex 15. In comparison, reduced efficiency was observed with catalyst $\mathbf{5}$, and similar observations were recorded for several other substrates.

Hydroamidation of several citronellol and prenol derivatives (e.g., product 24) revealed remarkable compatibility with acid-sensitive functional groups including silyl ethers (e.g., products 25 and 26), alkyl ethers (products 
27 and 28), $N$-Boc-protected amines (e.g., product 29), and acetals (products 30 and 32), including protected glycosides (product 31). Aliphatic aldehydes, ketones, halides, and alcohols were also found to be suitable substrates (products 33-36), including a labile tertiary benzhydryl derivative (product 37). Prenylated motifs containing nucleophilic functionalities properly positioned for intramolecular capture by the carbocationic intermediates also underwent successful hydroamidation, including aryl ethers (product 38) and indoles (products $\mathbf{3 9}$ and 40). ${ }^{21}$ Other trisubstituted alkenes demonstrated satisfactory reactivity under the conditions (products $\mathbf{4 1}$ and $\mathbf{4 2}$ ). Cyclic substrates also participated in the hydroamidation events (products 43-45), but a silylated bisabolol derivative underwent selective reaction at the terminal prenyl unit (product 46).

In all cases, formation of the desired acetamides was accompanied by varying amounts of the corresponding alcohols. Analysis of the hydroamidation and hydration products obtained from citronellyl acetate $(\mathbf{2 1})$ in the presence of $\mathrm{H}_{2}{ }^{18} \mathrm{O}$ revealed nearly identical extent of labelling $\left(92 \%\right.$ of $\left.{ }^{18} \mathrm{O}\right)$, suggesting that the alcohol arises primarily from the capture of carbocationic intermediates and not from radical reaction with adventitious molecular oxygen. ${ }^{22,23}$ The observed contribution of the hydration pathway was proportional to the excess of added water and could be attenuated accordingly when necessary (see product 42 in Table 2). Experiments with heavy water did not afford any deuterium-labelled products, arguing against the possibility of direct protonation of the alkene and in agreement with the proposed involvement of radical intermediates.

The difference in reactivity of cobalt salen complexes in the hydroamidation events (see Table 1) coupled with complete consumption of the silane and oxidant prompted us to investigate pathways that did not lead to apparent engagement of the alkene in HAT. We reasoned that formation of molecular hydrogen from the putative cobalt hydride intermediates, which was previously proposed to occur in HAT-initiated reactions, could contribute to the background reactivity. ${ }^{24-27}$ Our analysis of the headspace in the hydroamidation of alkene $\mathbf{6}$ and citronellyl acetate (21) and corresponding experiments in the absence of substrates revealed formation of $\mathrm{H}_{2}$ in all cases, but also highlighted striking differences in the behavior of cobalt complexes (Table 3). Catalysts $\mathbf{3}$ and $\mathbf{9}$ rapidly produced large quantities of molecular hydrogen both in the presence and absence of the substrates, pointing to significant contribution of this pathway to consumption of the silane in these cases. ${ }^{28}$ These observations were also consistent with the typical need for large excess of the corresponding reagents in relevant transformations. ${ }^{7,15}$ Complex 5 demonstrated a somewhat different reactivity profile, where production of $\mathrm{H}_{2}$ was significantly reduced in the presence of citronellyl acetate (21), suggesting direct competition of this pathway with the HAT to the alkene. No difference in formation of hydrogen was found in the
Table 3. Yield of Hydrogen Under the Conditions of the Catalytic Radical-Polar Crossover Ritter Reaction ${ }^{a}$

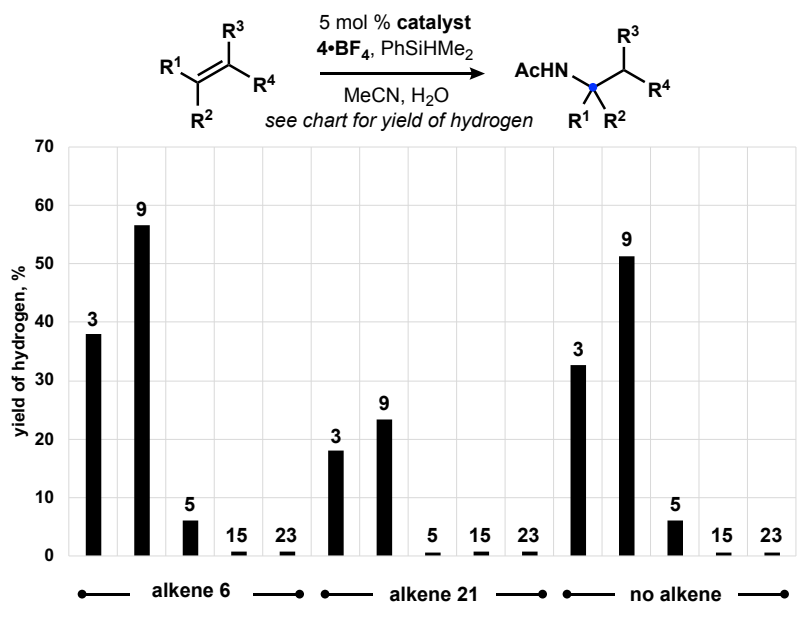

ayields of $\mathrm{H}_{2}$ were determined after $1 \mathrm{~h} 15 \mathrm{~min}$ by GCTCD analysis of the headspace in a closed vessel.

presence and absence of alkene $\mathbf{6}$, which was consistent with the lack of desired reactivity for catalyst 5 observed during the optimization studies. In contrast, only trace amounts of hydrogen were detected in the presence of catalysts $\mathbf{1 5}$ and $\mathbf{2 3}$ under all conditions examined. ${ }^{29}$

Taken together with complete consumption of the silane, our data demonstrate that background wasting of the reductant and oxidant catalyzed by the cobalt complexes involves production of $\mathrm{H}_{2}$, but is not limited to this pathway. These observations raise a possibility of contribution from catalytic processes that are not dependent on generation of the putative cobalt hydride intermediates. For example, collidinium radical cation, generated together with cobalt(III) fluoride upon oxidation of the cobalt(II) complex with $N$-fluorocollidinium salt, may react with the silane to generate the collidinium salt and a silyl radical (Scheme 5) ${ }^{30,31}$ Subsequent fluorine abstraction

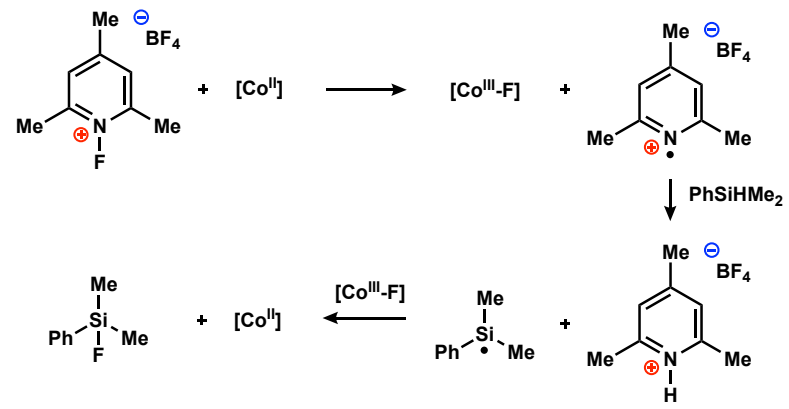

Scheme 5. Proposed mechanism for background consumption of the reductant and oxidant without formation of $\mathrm{H}_{2}$.

from the cobalt(III) fluoride by the silyl radical would complete the catalytic cycle. ${ }^{32,33}$ Irrespective of the mechanistic detail, the superior performance in the hydroamidation combined with minimal production of molecular hydrogen suggest that catalysts $\mathbf{1 5}$ and $\mathbf{2 3}$ offer improved partitioning of reaction pathways available to the putative cobalt hydride intermediates in favor of HAT to alkenes. 
In summary, we disclose a radical-polar crossover Ritter reaction that allows for hydroamidation of alkenes with diverse substitution patterns. In contrast to the original acid-catalyzed transformation, our process offers remarkable tolerance of functional groups. The structure of cobalt complexes plays key role in enabling a broad scope of substrates, where optimal catalysts overcome the commonly observed limitations and allow for efficient engagement of tri- and tetrasubstituted carbon-carbon double bonds. We demonstrate that formation of hydrogen contributes to consumption of the reductant and oxidant and competes with the desired hydroamidation events, suggesting a mechanistic link between HAT-initiated organic reactions and hydrogen evolution catalysis. ${ }^{34,35} \mathrm{We}$ also show that the ligand environment in a cobalt complex has a significant effect on the course of these background pathways, pointing to superior reactivity profile of putative cobalt hydride intermediates in the case of optimal catalysts. These studies can facilitate better understanding of intermediates involved in the HAT and identification of new catalysts with improved reactivity towards alkenes in other relevant hydrofunctionalizations.

\section{ASSOCIATED CONTENT}

Experimental procedures and characterization data for all new compounds.

\section{AUTHOR INFORMATION}

\section{Corresponding Author}

spronin@uci.edu

\section{Present Address}

${ }^{\dagger}$ Bristol Myers Squibb, New Brunswick, NJ 08901

\section{Author Contributions}

$\$$ These authors contributed equally. Notes

The authors declare no competing financial interest.

\section{ACKNOWLEDGMENT}

Financial support for this work was provided by the NSF (CAREER CHE-1848076). Acknowledgment is made to the Donors of the American Chemical Society Petroleum Research Fund for partial support of this research (PRF 58063DNI1). Funding from the School of Physical Sciences and the Chao Family Comprehensive Cancer Center is also gratefully acknowledged. E.E.T. is a recipient of Allergan Graduate Fellowship. We thank Sarah Wang and Professor Jenny Yang for providing access to their instrumentation and assistance with GC-TCD analysis. We also thank Professors Larry Overman, Chris Vanderwal, and Ryan Shenvi (TSRI) for helpful discussions.

\section{REFERENCES}

(1) Ritter, J. J.; Kalish, J. A new reaction of nitriles. II. Synthesis of $t$-carbinamines. J. Am. Chem. Soc. 1948, 70, 4048.

(2) Roose, P.; Eller, K.; Henkes, E.; Rossbacher, R.; Höke, H. Amines, aliphatic. Ullmann's Encyclopedia of Industrial Chemistry 2015, 1-55.
(3) Reider, P. J. Advances in AIDS chemotherapy: the asymmetric synthesis of CRIXIVAN ${ }^{\circledR}$. Chimia 1997, 51, 306.

(4) Guérinot, A.; Reymond, S.; Cossy, J. Ritter reaction: recent catalytic developments. Eur. J. Org. Chem. 2012, 2012, 19.

(5) George, D. T. Total synthesis of paxilline indole diterpenes and development of new chemical methods utilizing cobalt catalysis. Ph.D Dissertation, University of California, Irvine, CA, 2018.

(6) For excellent review of HAT-initiated reactions see: Crossley, S. W. M.; Martinez, R. M.; Obradors, C.; Shenvi, R. A. Mn, Fe, and Cocatalyzed radical hydrofunctionalizations of olefins. Chem. Rev. 2016 , 116,8912 .

(7) For proposed oxidation of alkyl radicals following the HAT in radical-polar crossover reactions see: (a) Shigehisa, H.; Aoki, T.; Yamaguchi, S.; Shimizu, N.; Hiroya, K. Hydroalkoxylation of unactivated olefins with carbon radicals and carbocation species as key intermediates. J. Am. Chem. Soc. 2013, 135, 10306. (b) Shigehisa, H.; Hayashi, M.; Ohkawa, H.; Suzuki, T.; Okayasu, H.; Mukai, M.; Yamazaki, A.; Kawai, R.; Kikuchi, H.; Satoh, Y.; Fukuyama, A.; Hiroya, K. Catalytic synthesis of saturated oxygen heterocycles by hydrofunctionalization of unactivated olefins: unprotected and protected strategies $J$. Am. Chem. Soc. 2016, 138, 10597.

(8) For proposed generation and participation of alkylcobalt(IV) intermediates in HAT-initiated radical-polar crossover reactions see: (a) Touney, E. E.; Foy, N. J.; Pronin, S. V. Catalytic radical-polar crossover reactions of allylic alcohols. J. Am. Chem. Soc. 2018, 140, 16982. (b) Discolo, C. A.; Touney, E. E.; Pronin, S. V. Catalytic asymmetric radical-polar crossover hydroalkoxylation. J. Am. Chem. Soc. 2019, $141,17527$.

(9) For dissociation of alkylcobalt(VI) intermediates to generate carbocations see: Samsel, E. G.; Kochi, J. K. Radical chain mechanism for alkyl rearrangement in organocobalt complexes. J. Am. Chem. Soc. 1986, 108,4790 .

(10) The relative contribution of these pathways is likely dependent on the substitution pattern of the alkenes. We favor intermediacy of carbocations en route to $N$-(tert-alkyl)amides due to sterically encumbered nature of alkylcobalt(IV) electrophiles.

(11) Waser, J.; Carreira, E. M. Convenient synthesis of alkylhydrazides by the cobalt-catalyzed hydrohydrazination reaction of olefins and azodicarboxylates. J. Am. Chem. Soc. 2004, 126, 5676.

(12) Waser, J.; Nambu, H.; Carreira, E. M. Cobalt-catalyzed hydroazidation of olefins: convenient access to alkyl azides. J. Am. Chem. Soc. 2005, 127, 8294 .

(13) Waser, J.; Gaspar, B.; Nambu, H.; Carreira, E. M. Hydrazines and azides via the metal-catalyzed hydrohydrazination and hydroazidation of olefins. J. Am. Chem. Soc. 2006, 128, 11693.

(14) Leggans, E. K.; Barker, T. J.; Duncan, K. K.; Boger, D. L. Iron(III) $/ \mathrm{NaBH}_{4}$-Mediated additions to unactivated alkenes: synthesis of novel 20'-vinblastine analogues. Org. Lett. 2012, 14, 1428.

(15) Shigehisa, H.; Koseki, N.; Shimizu, N.; Fujisawa, M.; Niitsu, M.; Hiroya, K. Catalytic hydroamination of unactivated olefins using a Co catalyst for complex molecule synthesis. J. Am. Chem. Soc. 2014 , 136, 13534.

(16) Yahata, K.; Kaneko, Y.; Akai, S. Cobalt-catalyzed intermolecular Markovnikov hydroamination of nonactivated olefins: $\mathrm{N}^{2}$-selective alkylation of benzotriazole. Org. Lett. 2020, 22, 598.

(17) While these investigations were in progress, a preprint describing related efforts appeared: Yin, Y.-N.; Ding, R.-Q.; Ouyang, D.-C.; Zhang, Q.; Zhu, R. Highly chemoselective synthesis of hindered Amides via cobalt-catalyzed intermolecular oxidative hydroamidation. ChemRxiv. Preprint. https://doi.org/10.26434/chemrxiv.13285484.v1.

(18) In all cases, complete consumption of the silane was observed.

(19) Observation of the same ratio of products $\mathbf{7}$ and $\mathbf{8}$ in all cases examined suggests that the corresponding carbocationic intermediates undergo rapid and reversible hydride shifts and are in equilibrium with each other

(20) For relevant hydrogenation see: (a) Iwasaki, K.; Wan, K. K.; Oppedisano, A.; Crossley, S. W. M.; Shenvi, R. A. Simple, chemoselective hydrogenation with thermodynamic control. J. Am. Chem. Soc. 2014, 136, 1300. (b) King, S. M.; Ma, X.; Herzon, S. B. A method for the selective hydrogenation of alkenyl halides to alkyl halides. J. Am. Chem. Soc. 2014, 136, 6884. 
(21) For relevant cyclizations see: (a) Crossley, S. W. M.; Barabé, F.; Shenvi, R. A. Simple, chemoselective, catalytic olefin isomerization. J. Am. Chem. Soc. 2014, 136, 16788. (b) Shigehisa, H.; Ano, T.; Honma, H.; Ebisawa, K.; Hiroya, K. Co-Catalyzed hydroarylation of unactivated olefins Org. Lett. 2016, 18, 3622. (c) Vrubliauskas, D.; Vanderwal, C. D. Cobalt-catalyzed hydrogen atom transfer induces bicyclizations that tolerate electron-rich and electron-deficient intermediate alkenes. Angew. Chem. Int. Ed. 2020, 59, 6115. (d) Vrubliauskas, D.; Gross, B. M.; Vanderwal, C. D. Stereocontrolled radical bicyclizations of oxygenated precursors enables short syntheses of oxidized abietane diterpenoids. J. Am. Chem. Soc. 2021, 143, 2944.

(22) For pioneering work on radical hydration see: Mukaiyama, T.; Yamada, T. Recent advances in aerobic oxygenation. Bull. Chem. Soc. Jpn. 1995, 68, 17 and references therein.

(23) See Supporting Information for details.

(24) (a) Bhattacharjee, A.; Chavarot-Kerlidou, M.; Andreiadis, E. S.; Fontecave, M.; Field, M. J.; Artero, V. Combined experimental-theoretical characterization of the hydrido-cobaloxime $\left[\mathrm{HCo}(\mathrm{dmgH})_{2}\left(\mathrm{P}^{n} \mathrm{Bu}_{3}\right)\right]$. Inorg. Chem. 2012, 51, 7087. (b) Estes, D. P.; Grills, D. C.; Norton, J. R. The reaction of cobaloximes with hydrogen: products and thermodynamics. J. Am. Chem. Soc. 2014, 136, 17362. (c) Lacy, D. C.; Roberts, G. M.; Peters, J. C. The cobalt hydride that never was: revisiting Schrauzer's "hydridocobaloxime". J. Am. Chem. Soc. 2015, 137, 4860 .

(25) Formation of $\mathrm{H}_{2}$ was previously proposed to accompany relevant HAT-initiated reactions: (a) Shevick, S. L.; Obradors, C.; Shenvi, R. A. Mechanistic interrogation of $\mathrm{Co} / \mathrm{Ni}$-dual catalyzed hydroarylation. J. Am. Chem. Soc. 2018, 140, 12056. (b) Matos, J. L. M.; Green, S. A.; Chun, Y.; Dang, V. Q.; Dushin, R. G.; Richardson, P.; Chen, J. S.; Piotrowski, D. W.; Paegel, B. M.; Shenvi, R. A. Cycloisomerization of olefins in water. Angew. Chem. Int. Ed. 2020, 59, 12998.

(26) For relevant discussion see: Shevick, S. L.; Wilson, C. V.; Kotesova, S.; Kim, D.; Holland, P. L.; Shenvi, R. A. Catalytic hydrogen atom transfer to alkenes: a roadmap for metal hydrides and radicals. Chem. Sci. 2020, 11, 12401 .

(27) For selected examples of leveraging a reverse reaction for generation of putative cobalt hydrides in HAT-initiated processes see: (a) Li, G.; Han, A.; Pulling, M. E.; Estes, D. P.; Norton, J. R. Evidence for formation of a Co-H Bond from $\left(\mathrm{H}_{2} \mathrm{O}\right)_{2} \mathrm{Co}\left(\mathrm{dmgBF}_{2}\right)_{2}$ under $\mathrm{H}_{2}$ : application to radical cyclizations. J. Am. Chem. Soc. 2012, 134, 14662. (b) Li, G.; Kuo, J. L.; Han, A.; Abuyuan, J. M.; Young, L. C.; Norton, J. R.; Palmer, J. H. Radical isomerization and cycloisomerization initiated by $\mathrm{H} \bullet$ transfer. J. Am. Chem. Soc. 2016, 138, 7698. See also ref. 24(b).

(28) Sampling of the headspace at different time points indicated comparable hydrogen content as early as $10 \mathrm{~min}$ after addition of silane.

(29) No hydrogen was detected in the absence of catalysts.

(30) For relevant considerations regarding generation of collidinium radical cation see: Shigehisa, H.; Nishi, E.; Fujisawa, M.; Hiroya, K. Cobalt-catalyzed hydrofluorination of unactivated olefins: a radical approach of fluorine transfer. Org. Lett. 2013, 15, 5158.

(31) Formation of silyl radicals was previously proposed in a relevant HAT-initiated hydrosilylation: Obradors, C.; Martinez, R. M.; Shenvi, R. A. $\mathrm{Ph}(i-\mathrm{PrO}) \mathrm{SiH}_{2}$ : an exceptional reductant for metal-catalyzed hydrogen atom transfers. J. Am. Chem. Soc. 2016, 138, 4962.

(32) Fluorine transfer from a cobalt fluoride complex to an alkyl radical intermediates was recently proposed: Guo, P.; Li, Y.; Zhang, X.G.; Han, J.-F.; Yu, Y.; Zhu, J.; Ye, K.-Y. Redox neutral radical-relay cobalt catalysis toward $\mathrm{C}-\mathrm{H}$ fluorination and amination. Org. Lett. 2020, 22, 3601.

(33) Formation of dimethylphenylfluorosilane was observed by GCMSD analysis in all cases.

(34) For examples of relevant studies in hydrogen evolution catalysis see: (a) Dempsey, J. L.; Brunschwig, B. S.; Winkler, J. R.; Gray, H. B. Hydrogen evolution catalysis by cobaloximes. Acc. Chem. Res. 2009, 42, 1995. (b) Jacques, P.-A.; Altero, V.; Pécaut, J.; Fontecave, M. Cobalt and nickel diimine-dioxime complexes as molecular electrocatalysts for hydrogen evolution with low overvoltages. Proc. Natl. Acad. Sci. USA 2009, 106, 20627.

(35) For examples of hydrogen evolution catalysis by relevant cobalt salen complexes see: Li, C.-B.; Gong, P.; Yang, Y.; Wang, H.-Y. Cobalt(II)-salen complexes for photocatalytic hydrogen production in noble metal-free molecular systems. Catal. Lett. 2018, 148, 3158. 